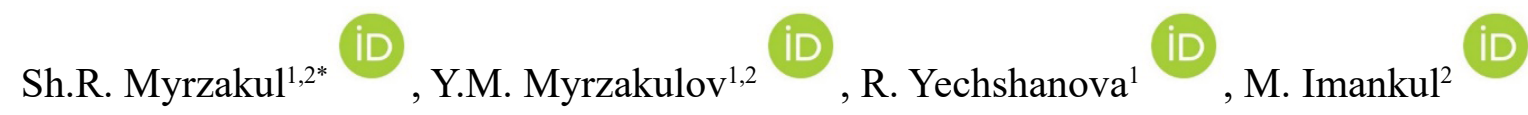 \\ ${ }^{1}$ L.N.Gumilyov Eurasian National University, Nur-Sultan, Kazakhstan \\ ${ }^{2}$ «Ratbay Myrzakulov Eurasian International Centre for Theoretical Physics» LLP, Nur-Sultan, Kazakhstan \\ *e-mail:srmyrzakul@gmail.com
}

\title{
Inflation in modified quantum gravity with a fermion field
}

\begin{abstract}
Viable inflation of modified nonuniform isotropic $F(R)$ gravity with a fermionic field of $f$-essence is investigated using the quantum approach. The action of which is $S=\int d^{4} x \sqrt{-g}[F(R)+$ $L_{m}$ ], where $R$ is the curvature scalar, and $L_{m}$ is the matter Lagrangian. In this case, we consider a non-minimally coupled fermionic field $f$-essence, the Lagrangian of which is denoted by $K(Y, u)$ by a function depending on Y-kinetic and $u$ potential arguments. The equations of motion of this model are obtained for the homogeneous and isotropic Friedman-Robertson-Walker space-time. As $F(R)$ we consider the generalized Horava-Lifshitz quantum gravity function. In 2009, Horava proposed a new approach to studying membranes in the theory of quantum gravity, known as the Horava-Lifshitz gravity.The peculiarity of Horava-Lifshitz gravity is that it is renormalizable. Further, the particular case of $K(Y, u)=\ln Y+u$ is investigated in detail. The parameters of describing the current accelerated expansion of the Universe are obtained and the explicit form of the connection of matter with space-time $h(u)$ is determined. The inflationary period of the evolution of this model is also investigated. To describe the inflationary period, the form of the Hubble parameter and the slow roll-off parameter, as well as other inflationary parameters, were determined. The presented results are compared with the observation results. The analysis of the results coincides with the observation data at certain values of the integral constants in the solutions.
\end{abstract}

Key words: $f$-essence, inflation, Friedman-Robertson-Walker metric, Horava-Lifshitz gravity, $F(R)$ gravity.

\section{Introduction}

At present, from observations of type Ia supernova explosions [1,2], it became known about the accelerated expansion of the Universe. Scientists believe that the reason for this phenomenon is the strength of the alleged hypothetical "dark energy". After the birth or the so-called Big Bang, our Universe also expanded rapidly. Scientists called this era inflation. However, there is still no unified theory describing these periods of the evolution of the Universe. In an attempt to create such a theory, scientists have proposed various models. These models can be divided into two main classes: this is a modification of Einstein's theory of relativity or the introduction of a previously unknown substance of "dark energy"

In addition, due to the difficulties of constructing a general theory взаимосвязьты unifies the two fundamental theories of general relativity and quantum mechanics, it also led to a modification of the theory of gravity. Meanwhile, it is assumed that matter fields (bosons and fermions) arise from superstructures (for example, Higgs bosons or superstrings), which, undergoing certain phase transitions, generate known particles. With that it is assumed that geometry (for example, the Ricci tensor or the Ricci scalar) interacts directly with the quantum fields of matter. This interaction necessarily modifies the standard theory of gravity, that is, the Lagrangian of gravity plus the effective fields are modified compared to the Einstein field.

Since 2009, the quantum theory of gravity by Horava-Lifshitz has established [3]. This approach is most likely manifested Based on this approach the influence of communication in the dimension of space-time. This approach takes into account the influence of relationships when assessing spatial and temporal characteristics. One of the main characteristics of this formulation is to abstain from specific Lorentz invariance so that it can be recreated when considering low energies as an 
approximate symmetry. Although this theory describing gravity is fundamental, some problems remain unanswered (for example [4-9]) related to detailed balance, with strong couplings, an extra spread degree of freedom of dissemination, the limit of general relativity (infrared), a relationship with alternative gravitational theories and other problems. Moreover, the investigation of Horava-Lifshitz gravity in the spatially flat Friedman-Robertson-Walker cosmology (FRW) determine that its describing cosmology [10] is analogical with ordinary general relativity. Although effective dark matter can act as a kind of integration constant in Horava-Lifshitz gravity. Consequently, there is no natural way (without additional fields) to obtain an accelerating Universe from Horava-Lifshitz gravity, let alone a unified description of early inflation with late time acceleration.

Inflation was proposed by Guth and Sato [11-13] several years ago to address the initial conditions of the Friedman universe, and today the idea is well accepted, according to which the Universe has undergone a period of strong accelerated expansion after the Big Bang. Although the arena of early acceleration models is quite large, the constraints that a theory must satisfy in order to reproduce the latest cosmological data $[14,15]$ are rather restrictive (see [16-20] for a general overview of inflation).

Inflation occurs in Planck time $\left(\sim 10^{-35 /-36}\right.$ seconds) and leads to thermalization of the observable Universe: as it accelerates expansion, the small initial velocities within the causal area become very large, and the horizon and flatness problems can be well explained. To cause acceleration, repulsive gravity is needed. All data indicate that inflation was realized with the help of the (quasi) de Sitter solution, but for the restoration of a Universe dominated by radiation / matter, a mechanism for a quick exit from the acceleration regime is necessary.

In this paper, we investigate the inflation of modified quantum gravity with a fermionic field. The generalized $F(R)$ Horava-Lifshitz gravity is considered as a modified quantum theory of gravity.

Model. Let's first examine the dynamics of the $f$-essence for the quantum modelof $F(R)$ gravity at FRW space-time

$$
d s^{2}=-c^{2} d t^{2}+a(t)^{2} \sum_{i=1}\left(d x^{i}\right)^{2},
$$

where $a(t)$ is the scale factor.

The action for $F(R)$ gravity is given as follows

$$
S=\int d^{4} x e\left[F(R)+L_{m}\right],
$$

here $e=\operatorname{det}\left(e_{\mu}^{i}\right)=\sqrt{-g}, R$ is the curvature scalar and $L_{m}$ is the matter Lagrangian. The generalized Horava-Lifshitz curvature proposed in [21] is considered as the curvature of space-time:

$$
R=3(1-3 \lambda+4 \mu) \frac{\dot{a}^{2}}{a^{2}}+6 \mu \frac{\ddot{a}}{a},
$$

where the dot means the time derivative.

According to [21], consider the action described above as a representation of a evalution system in which $a$ - scale factor and $R$ - scalar curvature are executed as independent non-stationary variables. Thus, action (2) can be rewritten as

$$
S=\int L(a, \dot{a}, R, \dot{R}) d t=\int\left\{a^{3} h(u) F(R)-v\left[R-\left(3(1-3 \lambda+4 \mu) \frac{\dot{a}^{2}}{a^{2}}+6 \mu \frac{\ddot{a}}{a}\right)+2 K(Y, u)\right]\right\} d t
$$

where $K(Y, u)$ is the Lagrangian of the $f$-essence, $u=\bar{\psi} \psi, \psi=\left(\psi_{0}, \psi_{1}, \psi_{2}, \psi_{3}\right)^{T}$ is a function of the fermionic field, and $\bar{\psi}=\psi^{+} \gamma^{0}$ denotes its conjugate function, $\quad v=a^{3} d F(R) / d R$ is the Lagrange multiplier and can be determined by changing the Lagrangian with respect to the scalar, which gives $v=a^{3} F^{\prime}$; in what follows, the curvature derivative $R$ will be denoted by a prime.
In addition, the kinetic part is determined by the following expression:

$$
Y=\frac{1}{2} i\left[\bar{\psi} \Gamma^{\mu} D_{\mu} \dot{\psi}-\left(D_{\mu} \dot{\bar{\psi}}\right) \Gamma^{\mu} \psi\right]
$$

here the differential operator $D_{\mu}$ is defined as the covariant derivative and $\Gamma^{\mu}=e_{i}^{\mu} \gamma^{i}$. Dirac matrices $\Gamma^{\mu}$ of curved space-time: 


$$
\begin{aligned}
& \Gamma^{0}=\gamma^{0}, \\
& \Gamma^{j}=a^{-1} \gamma^{j}, \\
& \Gamma^{5}=-i \sqrt{-g} \Gamma^{0} \Gamma^{1} \Gamma^{2} \Gamma^{3}=\gamma^{5}, \\
& \Gamma_{0}=\gamma^{0}, \\
& \Gamma_{j}=a \gamma^{j}, \quad(i=1,2,3),
\end{aligned}
$$

where the gamma matrices are of the form

$$
\begin{gathered}
\gamma^{0}=\left(\begin{array}{cc}
I & 0 \\
0 & -I
\end{array}\right), \quad \gamma^{k}=\left(\begin{array}{cc}
0 & \sigma^{k} \\
-\sigma^{k} & 0
\end{array}\right), \\
\gamma^{5}=\left(\begin{array}{ll}
0 & I \\
I & 0
\end{array}\right),
\end{gathered}
$$

where $I=\operatorname{diag}(1,1)$ and $\sigma^{k}$ are Pauli matrices, defined as

$$
\begin{gathered}
\sigma^{1}=\left(\begin{array}{ll}
0 & 1 \\
1 & 0
\end{array}\right), \quad \sigma^{2}=\left(\begin{array}{cc}
0 & -i \\
i & 0
\end{array}\right), \\
\sigma^{3}=\left(\begin{array}{cc}
1 & 0 \\
0 & -1
\end{array}\right) .
\end{gathered}
$$

Then the kinetic part for the FRW metric takes the form

$$
Y=\frac{1}{2} i\left(\bar{\psi} \gamma^{0} \dot{\psi}-\dot{\bar{\psi}} \gamma^{0} \psi\right)
$$

Finally, the effective Lagrangian from action (4) has the form

$$
\begin{gathered}
L=a^{3} h F+h F^{\prime} a^{3} R-3(1-3 \lambda) \dot{a}^{2} a h F^{\prime}+ \\
+6 \mu \dot{a} a^{2} h_{u} \dot{u} F^{\prime}+6 \mu \dot{a} a^{2} h F^{\prime \prime} \dot{R}+2 a^{3} K,
\end{gathered}
$$

subscripts denote derivatives with respect to the given parameters.

Using the Euler-Lagrange method to (10), we obtain the equations of motion for the spinor field and the complex field conjugate to

$$
\begin{gathered}
\dot{\bar{\psi}}+\frac{1}{2}\left(3 H+\frac{\dot{K}_{Y}}{K_{Y}}\right) \bar{\psi}-i \frac{K_{u}}{K_{Y}} \bar{\psi} \gamma^{0}- \\
-\frac{i}{2}\left[\frac{F}{F^{\prime}}-R+6\left(2 H^{2}+\dot{H}\right)\right] \frac{F h_{u}}{K_{Y}} \bar{\psi} \gamma^{0}=0 \\
\dot{\psi}+\frac{1}{2}\left(3 H+\frac{\dot{K}_{Y}}{K_{Y}}\right) \psi+i \frac{K_{u}}{K_{Y}} \gamma^{0} \psi+ \\
+\frac{i}{2}\left[\frac{F}{F^{\prime}}-R+6\left(2 H^{2}+\dot{H}\right)\right] \frac{F h_{u}}{K_{Y}} \gamma^{0} \psi=0
\end{gathered}
$$

where $H=\frac{\dot{a}}{a}$ Hubble parameter.

Next, we obtain the FRW equations for pressure

$$
-\left(2 \dot{H}+3 H^{2}\right)=p_{f},
$$

$$
3(1-3 \lambda+6 \mu) \frac{\dot{a}^{2}}{a^{2}} H^{2}+6 \mu \dot{H}=R .
$$

From the conservation of energy

$$
E_{L}=\frac{\partial L}{\partial \dot{a}} \dot{a}+\frac{\partial L}{\partial \dot{\bar{\psi}}} \dot{\bar{\psi}}+\frac{\partial L}{\partial \dot{\psi}} \dot{\psi}-L=0,
$$

one can obtain the FRW equation for the energy density

$$
3 H^{2}=\rho_{f} \text {. }
$$

The expressions for the energy density $\rho_{f}$ and the pressure $p_{f}$ of the fermion field have the form

$$
\begin{gathered}
\rho_{f}=\frac{1}{1-3 \lambda} \frac{F}{F^{\prime}}-\frac{1}{1-3 \lambda} R+\frac{2}{1-3 \lambda} \frac{1}{F^{\prime}} \frac{K}{h}+ \\
+\frac{6 \mu}{1-3 \lambda} H\left(\frac{F^{\prime \prime}}{F^{\prime}} \dot{R}+\frac{\dot{h}}{h}\right)-\frac{2}{1-3 \lambda} \frac{K_{Y} Y}{h F^{\prime}}
\end{gathered}
$$

$$
\begin{gathered}
p_{f}=-\frac{1}{1-3 \lambda} \frac{F}{F^{\prime}}-\frac{2}{1-3 \lambda} \frac{1}{F^{\prime}} \frac{K}{h}-\frac{2 \mu}{1-3 \lambda} \times \\
\times\left(\frac{F^{\prime \prime \prime}}{F^{\prime}} \dot{R}^{2}+\frac{F^{\prime \prime}}{F^{\prime}} \ddot{R}+2 \frac{\dot{h}}{h} \frac{F^{\prime \prime}}{F^{\prime}} \dot{R}+\frac{\ddot{h}}{h}\right)+2 H\left(\frac{F^{\prime \prime}}{F^{\prime}} \dot{R}+\frac{\dot{h}}{h}\right) .
\end{gathered}
$$


To solve the system of equations of motion, we first consider equations (11) and (12). We multiply equation (11) by $\gamma^{0} \psi$ and equation (12) by $\gamma^{0} \bar{\psi}$, then summing these equations, we get the expression:

$$
\frac{\dot{u}}{u}=-3 H-\frac{\dot{K}}{K_{Y}},
$$

whose solution has the form

$$
u=\frac{u_{0}}{a^{3} K_{Y}}
$$

where $u_{0}$ is the constant of integration. Next, we get differential equality with separated variables, which will be equal to the constant

$$
\begin{gathered}
\frac{-2 K_{Y} Y-2 K_{u} u}{h_{u} K_{u}}= \\
=F^{\prime}\left(\frac{F}{F^{\prime}}-R+3(1-3 \lambda+6 \mu) H^{2}+6 \mu \dot{H}\right)=C .
\end{gathered}
$$

Consider the $f$-essence Lagrangian in the form

$$
K=\ln Y+u,
$$

substituting into the left-hand side of equation (21), we obtain the connection of matter with space-time as

$$
h=\frac{u}{C}+\frac{u^{2}}{2 C}+C_{1}
$$

then dividing the variables on the right-hand side of the equation, we obtain the equality

$$
\begin{aligned}
F-R F^{\prime}-\frac{C}{F^{\prime}}=- & {\left[3(1-3 \lambda+6 \mu) H^{2}+6 \mu \dot{H}\right]=} \\
= & G=\text { const },
\end{aligned}
$$

then the solution

$$
H^{2}=\frac{e^{-\frac{6(1-3 \lambda+4 \mu)}{6 \mu}\left(t-t_{0}\right)}-G}{3(1-3 \lambda+4 \mu)},
$$

that is, the Hubble parameter changes exponentially as

$$
H \sim e^{-\gamma\left(t-t_{0}\right)},
$$

where $\gamma=\frac{3(1-3 \lambda+4 \mu)}{6 \mu}$, hence the scale factor will take the form

$$
a=e^{e^{\gamma\left(t-t_{0}\right)}},
$$

also, from equation (24), the following dependence can be determined

$$
F(R) \sim R+R_{0} .
$$

Inflation. Consider an inflationary model with a minimum kinetic term, in which the behavior of the system is described by the FRU and Dirac equations. To describe evolution, it is convenient to introduce the functions of the Hubble flow, which are determined by the formula

$$
\varepsilon_{n+1} \equiv-\frac{d \ln \left|\varepsilon_{n}\right|}{\mathrm{d} N}, n \geq 0,
$$

where $\varepsilon_{0} \equiv H_{\mathrm{i} i n} / H \quad$ is the slow roll parameter. By definition, inflation is a phase of accelerated expansion $\ddot{a} / a>0$ or $\varepsilon_{1}<1$, which is the same. The de Sitter quasi solution of inflation evolves with (positive) Hubble flow functions

$$
\varepsilon_{1}=-\frac{\dot{H}}{H^{2}}, \quad \varepsilon_{2}=-\frac{2 \dot{H}}{H^{2}}+\frac{\ddot{H}}{H \dot{H}} \equiv \frac{\dot{\varepsilon}_{1}}{H \varepsilon_{1}},
$$

which should remain small until the end of inflation, when the acceleration ends $\varepsilon_{1} \cong 1 \quad(\dot{H}<0)$, therefore, in the model under study, the parameters of the Hubble flow have the same form

$$
\varepsilon_{1}=\varepsilon_{2}=e^{\gamma\left(t-t_{0}\right)}
$$

Also consider the number of e -folds remaining until the end of inflation

$$
N=\ln \left[\frac{a\left(t_{\mathrm{f}}\right)}{a(t)}\right],
$$


where $a\left(t_{\mathrm{i}}\right), a\left(t_{\mathrm{f}}\right) \quad 80 / 5000$ are scale factors at the beginning and at the end of inflation, respectively. The total number of inflation is determined as follows

$$
\mathrm{N} \equiv \ln \left(\frac{a_{\mathrm{f}}\left(t_{\mathrm{f}}\right)}{a_{\mathrm{i}}\left(t_{\mathrm{i}}\right)}\right)=\int_{t_{\mathrm{i}}}^{t_{\mathrm{f}}} H(t) d t
$$

and in order to have thermalization at the end of inflation, we must require, according to the $\mathrm{CMB}$ fluctuation spectrum, $55<\mathrm{N}<65$. Thus, the spectral index $n_{s}$ and the tensor-scalar relation $r$ are derived from the expressions below

$$
n_{s}=1-2 \varepsilon_{1}-\varepsilon_{2}, \quad r=16 \varepsilon_{1},
$$

where $\varepsilon_{1,2}$ are evaluated to $N=\mathrm{N}$. The latest cosmological data from the Planck satellite [15] limit these indices as follows: $n_{\mathrm{s}}=0.9603 \pm 0.0073(68 \% \mathrm{CL})$ and $r<0.11(95 \% \mathrm{CL})$.

\section{Conclusion}

This article discusses a complex gravity model within the framework of the Horava-Lifshitz theory for the f-entity. Studies have shown that when considering the Lagrangian (22) f-essence, the scale factor of this model grows exponentially (27), which indicates an accelerated expansion of the Universe. Compared to de Sitter's standard solution, this gives the universe a much faster acceleration. A linear dependence of the function on the scalar was also found. The results obtained coincide with the data of current observations.

\section{Acknowledgement}

The research work is supported by the grant project AP08052197 (2020-2022) from the Ministry of Science and Education of the Republic of Kazakhstan.

\section{References}

1 Riess, A. G. et al. "Observational Evidence from Supernovae for an Accelerating Universe and a Cosmological Constant." The Astrophysical Journal 116, no 3. (1998): 1009-1038.
2 Perlmutter, S. et al. "Measurements of $\Omega$ and $\Lambda$ from 42 High-Redshift Supernovae." The Astrophysical Journal 517, no. 2. (1999): 565-586.

3 Horava, P. "Quantum gravity at a Lifshitz point." Physical Rewiew D Vol.79, no. 8. (2009): 084008.

4 Capozziello, S., Cardone, V.F., and Troisi, A. "Dark energy and dark matter as curvature effects?." Journal of Cosmology and Astroparticle Physics, Vol.2006 (2006): 001.

5 Nojiri, S. and Odintsov, S.D. "Modified Gravity with $\ln R$ Terms and Cosmic Acceleration." General Relativity and Gravitation Vol.36 (2004): 1765-1780.

6 Capozziello, S., Cardone V.F., and Troisi A. "Reconciling dark energy models with $f(R)$ theories." Physical Review D Vol.71, no. 4. (2005): 043503.

$7 \mathrm{Li}$, B. and Barrow, J.D. "Cosmology of $f(R)$ gravity in the metric variational approach." Physical Review D Vol.75, no. 8. (2007): 084010.

8 Bean, R., Bernat, D., Pogosian, L., Silvestri, A., and Trodden, M. "Dynamics of linear perturbations in $f(R)$ gravity." Physical Review $D$, Vol.75, no. 6. (2007): 064020.

9 Navarro, I. and Van Acoleyen, K. " $f(R)$ actions, cosmic acceleration and local tests of gravity." Journal of Cosmology and Astroparticle Physics, Vol.2007 (2007): 022.

10 Stelle, K.S. "Classical gravity with higher derivatives." General Relativity and Gravitation Vol.9. (1978): 353-371.

11 Sato, K., "First Order Phase Transition of a Vacuum and Expansion of the Universe." Monthly Notices of the Royal Astronomical Society, Vol.195 (1981): 467-479.

12 Sato, K., "Cosmological baryon-number domain structure and the first order phase transition of a vacuum." Physics Letters B Vol.99, no. 1 (1981): 6670.

13 Guth, A.H., "Inflationary universe: A possible solution to the horizon and flatness problems." Physical Review D Vol.23, no. 2. (1981): 347.

14 Ade, P.A.R. et al. "Planck 2013 results. XVI. Cosmological parameters." Astronomy \& Astrophysics Vol.571 (2014): A16.

15 Komatsu, E. et al. "Five-Year Wilkinson Microwave Anisotropy Probe* Observations: Cosmological Interpretation". The Astrophysical Journal Supplement Series Vol.180, no. 2 (2009): 330-376. 
16 Myrzakul Sh., Myrzakulov K., Bekov S., Myrzakul T, Myrzakulov R. Starobinsky model with $f$-essence // [arXiv:1710.08413].

17 Jamil, M., Saridakis, E.N., and Setare M.R. "Holographic dark energy with varying gravitational constant." Physics Letters B Vol.679, no. 3. (2009): 172-176.

18 Mukohyama, Sh. "Dark matter as integration constant in Horava-Lifshitz gravity." Physical Review D Vol.80, no. 6 (2009): 064005.
19 Gorbunov, D.S. and Rubakov, V.A., Introduction to the Theory of the Early Universe: Hot Big Bang Theory. World Scientific, 2011.

20 Linde, A.D. "Inflationary Cosmology." Lecture Notes in Physics Vol.738 (2008): 1-54.

21 Chaichian M., Nojiri, S., Odintsov, S.D., Oksanen, M., and Tureanu A. "Modified $F(R)$ Horava-Lifshitz gravity: a way to accelerating FRW cosmology." Classical and Quantum Gravity Vol. 27, no. 18 (2010): 185021. 